\title{
Ptychographic image reconstruction using total variation regularization
}

\author{
R. Nebling, I. Mochi, H. Kim, A. Dejkameh, T. Shen, M. Guizar-Sicairos and Y. Ekinci \\ Paul Scherrer Institut, Villigen, Switzerland
}

ricarda.nebling@psi.ch

Coherent diffraction imaging (CDI) allows for sub-wavelength spatial resolution by reconstructing an image from recorded diffraction patterns using a phase-retrieval algorithm [1,2]. CDI is particularly advantageous in the extreme ultraviolet (EUV) and X-ray ranges, where optics manufacturing is difficult and expensive [2]. Ptychography, the scanning version of CDI, has several benefits, such as large field of view imaging and robustness. The sample (object) is scanned by moving a spatially confined illumination (probe) while ensuring overlap in the illuminated regions [3]. The complex object function is typically retrieved by using an iterative algorithm that relies on two constraints [4]. First, the real space (or overlap) constraint assumes that the exit wave leaving the sample is formed by the probe function multiplied with the object function, i.e. the thin object approximation [3]. Second, the Fourier constraint enforces the estimated diffraction pattern intensity to match the measured diffraction data.

To optimize the reconstruction procedure, additional constraints have been suggested, based on a priori knowledge of the object and the measurement system. For example, Guizar-Sicairos et al. [5] introduced a nonlinear optimization approach to solve the ptychographic problem, or Thibault et al. [6] proposed a statistical optimal reconstruction procedure that finds the solution by a leastsquares approximation of the maximum likelihood function. Alternatively, an approach by Katkovnik et al. [7] uses a sparse approximation of the probe and object function additionally to a maximum likelihood technique, to improve the reconstruction quality compared to a non-optimized algorithm. Recently, Ansuinelli et al. [8] have directly used the sample's layout information to build an optimal reconstruction algorithm for imaging a photolithography mask, by penalizing the deviation of the reconstructed mask image to a full mask model.

We present here a phase-retrieval algorithm similar to Chang et al. [9] and Enfedaque et al. [10] that solves the blind ptychography problem (retrieving the probe and object) using total variation regularization (TV) as an additional constraint on the object function. TV promotes a sparse object gradient and is therefore preferential for (quasi) binary structures, removing noise and image artefacts [11]. We will discuss the total variation based algorithm for EUV photolithography mask inspection and show the impact of the algorithm for reconstruction of simulated and experimental data.

[1] Chapman, H. N. \& Nugent, K. A. (2010). Nature Photonics, 12, pp. 833 - 830.

[2] Gardner, D., Tanksalvala, M., Shanblatt, E.R., et al. (2017). Nature Photonics, 11, pp. 259 - 263.

[3] Rodenburg, J. \& Faulkner, H. (2004). Appl. Phys. Lett., 85, pp. 4795 - 4797.

[4] Thibault, P., Dierolf, M., Bunk, O., et al. (2009). Ultramicroscopy, 109, pp. 338 - 343.

[5] Guizar-Sicairos, M. \& Fienup, J. (2008). Optics Express, 16, pp. 7264 - 7282.

[6] Thibault, P. \& Guizar-Sicairos, M. (2012). New Journal of Physics, 14, 063004.

[7] Katkovnik, V. \& Astola, J. (2012). J. Opt. Soc. Am. A, 30, pp. 367 - 379.

[8] Ansuinelli, P., Coene, W. M. J. \& Urbach, H. P. (2020). Applied Optics, 59, pp. 5937 - 5947.

[9] Chang, H., Enfedaque, P. \& Marchesini, S. (2019). 2019 IEEE International Conference on Image Processing, pp. 2931 - 2935.

[10] Enfedaque, P., Chang, H., Krishnan, H., et al. (2018). Computational Science - ICCS 2018, pp. 540 - 553.

[11] Rudin, L. I., Osher, S. \& Fatemi, E. (1992). Physica D, 60, pp. 259 - 268.

Keywords: ptychography; a priori information; phase-retrieval algorithm; total variation; EUV mask inspection;

Part of this research was performed at Swiss Light Source, Paul Scherrer Institut. Co-authors (A.T and R.N.) thank Swiss National Science Foundation for financial support (SNF Grant Number: 200021_172768). 